\title{
COVID-19 e a abordagem One Health (Saúde Única): uma revisão sistemática
}

\section{COVID-19 and the One Health approach: a systematic review}

\section{Jean Ezequiel Limongi',* (iD) \\ Stefan Vilges de Oliveira" (ID)}

I Instituto de Geografia, Universidade Federal de Uberlândia, Uberlândia, MG, Brasil

" Departamento de Saúde Coletiva, Faculdade de Medicina,Universidade Federal de Uberlândia, Uberlândia, MG, Brasil

\section{* E-mail: jeanlimongi@gmail.com}

Recebido: 14 maio 2020 Aprovado: 22 maio 2020

\section{RESUMO}

Introdução: Em dezembro de 2019, foi identificado em Wuhan, China, em pessoas que frequentavam um mercado úmido, a presença de um novo coronavírus, denominado SARS-CoV-2, responsável pela COVID-19, que rapidamente se espalhou pelo mundo de forma pandêmica. A história natural da doença se adapta de forma emblemática na abordagem One Health (Saúde Única), que são estratégias de controle de doenças que buscam de forma mais ampla discutir os aspectos indissociáveis da saúde humana, animal e ambiental. Objetivo: Realizar uma revisão sistemática de literatura sobre a aplicação dos conceitos de Saúde Única no atual contexto pandêmico da COVID-19. Método: 0 estudo seguiu a recomendação PRISMA para elaboração de revisões sistemáticas. As bases de dados pesquisadas foram PubMed, Scopus, Web of Science, Lilacs e Google Scholar. Os termos de pesquisa utilizados foram (COVID-19 OR SARS-CoV-2 OR 2019-nCoV) AND "One Health". Resultados: Na síntese qualitativa foram incluídas 62 publicações, 28 (45,2\%) apresentavam propostas para a vigilância em saúde de COVID-19 por meio de abordagem One Health e $34(54,8 \%)$ apenas relataram a importância dessa abordagem no combate ao vírus. Foi observado um incremento das publicações sobre os dois temas ao longo dos meses analisados. Conclusões: Questões biológicas (investigações relacionadas ao vírus, aos hospedeiros e à biotecnologia) foram os temas mais discutidos como estratégias de One Health, em detrimento das questões sociais, econômicas e comportamentais. Políticas governamentais devem ser adotadas para garantir as ações multiprofissionais de saúde. Cabe também aos profissionais a promoção e a aplicação deste conceito, de forma articulada e interdisciplinar. A aplicabilidade da One Health no contexto pandêmico vivenciado pela COVID-19 é imperiosa.

PALAVRAS-CHAVE: COVID-19; One Health; Revisão Sistemática

\section{ABSTRACT}

Introduction: In December 2019, was identified in Wuhan, China, the presence of a new coronavirus, called SARS-CoV-2, responsible for COVID-19, which quickly spread throughout the world in a pandemic manner. The natural history of the disease adapts itself in an emblematic way in the One Health approach, which are disease control strategies that seek more broadly to discuss the inseparable aspects of human, animal and environmental health. Objective: The aim of the study was to conduct a systematic review of the literature on the application of the concepts of One Health in the current pandemic context of COVID-19. Method: The study followed the PRISMA guidelines for the preparation of systematic reviews. The databases searched were PubMed, Scopus, Web of Science, Lilacs and Google Scholar. The search terms used were (COVID-19 OR SARS-CoV-2 OR 2019-nCoV) AND “One Health". Results: In the qualitative synthesis, 62 publications were included, 28 (45.2\%) presented proposals for COVID-19 health surveillance through the One Health approach and 34 (54.8\%) only reported the importance of this approach in controlling the virus. There was an increase in publications on the two themes over the months analyzed. Conclusions: Biological issues (investigations related to viruses, hosts and biotechnology) were the most discussed topics as One Health strategies, to the 
detriment of social, economic and behavioral issues. Government policies must be adopted to guarantee multiprofessional health actions. It is also up to professionals to promote and apply this concept, in an articulated and interdisciplinary way. The applicability of One Health in the pandemic context experienced by COVID-19 is imperative.

KEYWORDS: COVID-19; One Health; Systematic Review

\section{INTRODUÇÃO}

Os coronavírus (CoV) são um grupo de vírus de RNA de fita simples envelopados, que podem infectar pessoas e animais. Estes pertencem à subfamília Orthocoronavirinae, família Coronaviridae, na ordem Nidovirales' ${ }^{1}$. Eles estão classificados em quatro gêneros: Alfa, Beta, Gama e Deltacoronavírus. Os dois primeiros gêneros podem infectar humanos e estes patógenos apresentam distribuição global'. Nos animais, estes CoV podem produzir doença respiratória, gastrointestinal, hepática e neurológica ${ }^{2,3}$.

Já foram identificados sete CoV patogênicos para humanos ${ }^{2}$. Os sintomas mais frequentemente encontrados são de um resfriado comum e para maioria destes agentes etiológicos, raramente, ocorre a infecção grave do trato respiratório inferior, como pneumonia, principalmente em crianças, idosos e pacientes imunocomprometidos ${ }^{4,5}$.

Nas últimas duas décadas foram observados três episódios de transbordamento zoonótico - quando um agente etiológico de origem animal passa a infectar humanos - envolvendo $\mathrm{CoV}^{3,6}$. Estes foram importantes pela sua letalidade, magnitude e transcendência, quando trouxeram grande impacto aos serviços de saúde do planeta ${ }^{3}$.

O primeiro CoV a causar epidemia foi a Sars-CoV-1, registrado em 2002 como o agente etiológico de uma Síndrome Respiratória Aguda Grave (SARS), registrada inicialmente em Guangzhou (Guangdong, China), causando 8.422 casos e 916 mortes em 29 países nos cinco continentes ${ }^{7}$.

Dez anos após a SARS, outro CoV altamente patogênico surgiu nos países do Oriente Médio como o causador da Síndrome Respiratória do Oriente Médio (MERS). Um total de 2.494 casos foi confirmado, incluindo 858 mortes. Os casos foram relatados globalmente, mas a maioria desses foi registrado na Arábia Saudita ${ }^{8}$.

Em dezembro de 2019, foi identificado em Wuhan, China, em pessoas que frequentaram um mercado úmido, a presença de um novo CoV, denominado SARS-CoV-2, responsável pelaCOVID-19, que rapidamente se espalhou pelo mundo de forma pandêmica ${ }^{5}$.

Em comum aos três episódios, as investigações epidemiológicas mostraram que a emergência dos patógenos se deu pelo transbordamento zoonótico, no qual estes CoV, que anteriormente infectavam animais silvestres, passaram a infectar humanos, causando doenças graves de forma epidêmica ${ }^{3,6}$.Também foi observado que estes escapes zoonóticos foram impulsionados pelas práticas culturais da utilização de animais silvestres - morcegos, cobras, civetas, pangolins - como parte da dieta e como iguaria na alimentação das populações da Ásia ${ }^{1,3}$. Associadas a este aspecto estão as frágeis leis sanitárias e regulatórias destes países para a comercialização e fiscalização da produção e venda de animais silvestres que são os propagadores destes patógenos ${ }^{1,3}$.
Nesse sentido, surge a One Health (Saúde Única) que, de forma mais ampla, busca discutir os aspectos indissociáveis da saúde humana, animal e ambiental ${ }^{9}$. De acordo com essa abordagem, existem quatro áreas que influenciam na situação sanitária de um determinado território: o ambiente, as questões sociais, os aspectos econômicos e os comportamentais. Esse enfoque colaborativo entende que o estado sanitário dos seres humanos está relacionado com a saúde dos animais e que ambas as populações (homens e animais) afetam o ambiente que coexistem e são igualmente afetados por esse ambiente ${ }^{9}$. Essa compreensão mais ampla das situações de saúde tem possibilitado a adoção de estratégias mais efetivas sobre os determinantes de saúde-adoecimento-cuidado nos âmbitos dos serviços de saúde?. Entre as premissas da atuação da One Health estão a abordagem multiprofissional e as colaborações interdisciplinares, buscando o benefício dos cuidados de saúde, em seus aspectos mais amplos, para seres humanos, animais e o meio ambiente ${ }^{9}$.

Buscando realizar uma avaliação das estratégias da One Health que estão sendo adotadas e propostas no âmbito global para o enfrentamento da pandemia da COVID-19, este estudo teve por objetivo realizar uma revisão sistemática de literatura sobre a aplicação dos conceitos de Saúde Única no atual contexto pandêmico da COVID-19.

\section{MÉTODO}

\section{Protocolo de revisão sistemática}

As diretrizes e procedimentos dos Principais Itens para Relatar Revisões sistemáticas e Meta-análises (PRISMA) foram seguidas no presente estudo ${ }^{10}$. Não foi utilizado nenhum protocolo para avaliar a qualidade das publicações incluídas no estudo.

\section{Estratégia de busca e critério de elegibilidade}

Publicações que relatavam sobre a pandemia da COVID-19 e a abordagem One Health como estratégia de vigilância em saúde foram pesquisadas até o dia 2 de maio de 2020. Artigos originais, artigos de opinião, editoriais, revisões, cartas aos editores, comentários, comunicações curtas, perspectivas, relatos especiais e entrevistas foram incluídos na revisão sistemática. Além das publicações com revisão por pares, preprints também foram considerados. Foram pesquisadas cinco bases de dados, incluindo PubMed, Scopus, Web of Science, Literatura Latino-Americana e do Caribe em Ciências da Saúde (Lilacs) e Google Scholar. As bases de dados foram pesquisadas por um dos pesquisadores usando a variação dos termos de pesquisa 
(COVID-19 OR SARS-CoV-2 OR 2019-nCoV) AND “One Health". Artigos em outro idioma que não o inglês e o português foram excluídos desta revisão sistemática. Considerando a data dos primeiros relatos confirmados de casos da COVID-19, as buscas foram limitadas a artigos publicados em 2020.0s artigos que apresentavam a abordagem One Health e o novo coronavírus foram selecionados para serem analisados neste estudo.

\section{Rastreamento dos dados}

Foi realizada uma pesquisa no banco de dados e as publicações foram acessadas. 0 rastreamento ocorreu da seguinte forma:

- Apenas publicações que relatavam abordagem One Health e o novo coronavírus foram incluídos;

- Os resumos das publicações armazenadas foram lidos e, se necessário, as seções de introdução e/ou resultados e discussão foram cuidadosamente investigadas para garantir que as publicações atendessem aos critérios de inclusão;

- Dois revisores independentes realizaram a triagem dos títulos e resumos de acordo com os critérios de elegibilidade. Discordâncias foram discutidas e posteriormente resolvidas por consenso;

- Todos as publicações identificadas foram revisadas. As referências de cada publicação foram pesquisadas manualmente na busca de outros estudos de interesse desta revisão.

\section{Extração dos dados e síntese}

As seguintes categorias de dados foram coletadas: autoria, título da publicação, título da revista, tipo de publicação, data da publicação e propostas para a vigilância em saúde da COVID-19 baseadas na abordagem One Health. Aquelas publicações que não propunham estratégias específicas do tipo One Health, mas relatavam a importância delas no combate à COVID-19, foram coletadas e apresentadas à parte. Um dos revisores realizou a extração de dados e o outro revisor avaliou a precisão dos dados extraídos.

\section{RESULTADOS}

Foram excluídas as duplicatas entre as bases de dados $(n=21)$, e o procedimento de buscas eletrônicas recuperou 480 publicações. Após a leitura dos títulos e resumos, 87 textos foram conduzidos para leitura integral. Com 25 exclusões (não discutiram diretamente a relação da COVID-19 e a abordagem One Health: $n=5$; os termos COVID-19 e One Health constavam apenas na afiliação dos autores ou nas referências da publicação: $n=20$ ), 62publicações foram consideradas para elaboração da síntese qualitativa (Figura 1).

Os tipos de publicações encontrados foram variados, quais sejam: $18(29,1 \%)$ editoriais, $13(21,0 \%)$ artigos originais, nove $(14,5 \%)$ cartas aos editores, sete $(11,3 \%)$ artigos de revisão, seis $(9,7 \%)$ comentários, três $(4,8 \%)$ comunicações curtas, dois $(3,2 \%)$ artigos de opinião, duas $(3,2 \%)$ perspectivas, um $(1,6 \%)$ relato especial e uma $(1,6 \%)$ entrevista. Foram encontrados autores de 42 países representando cinco continentes

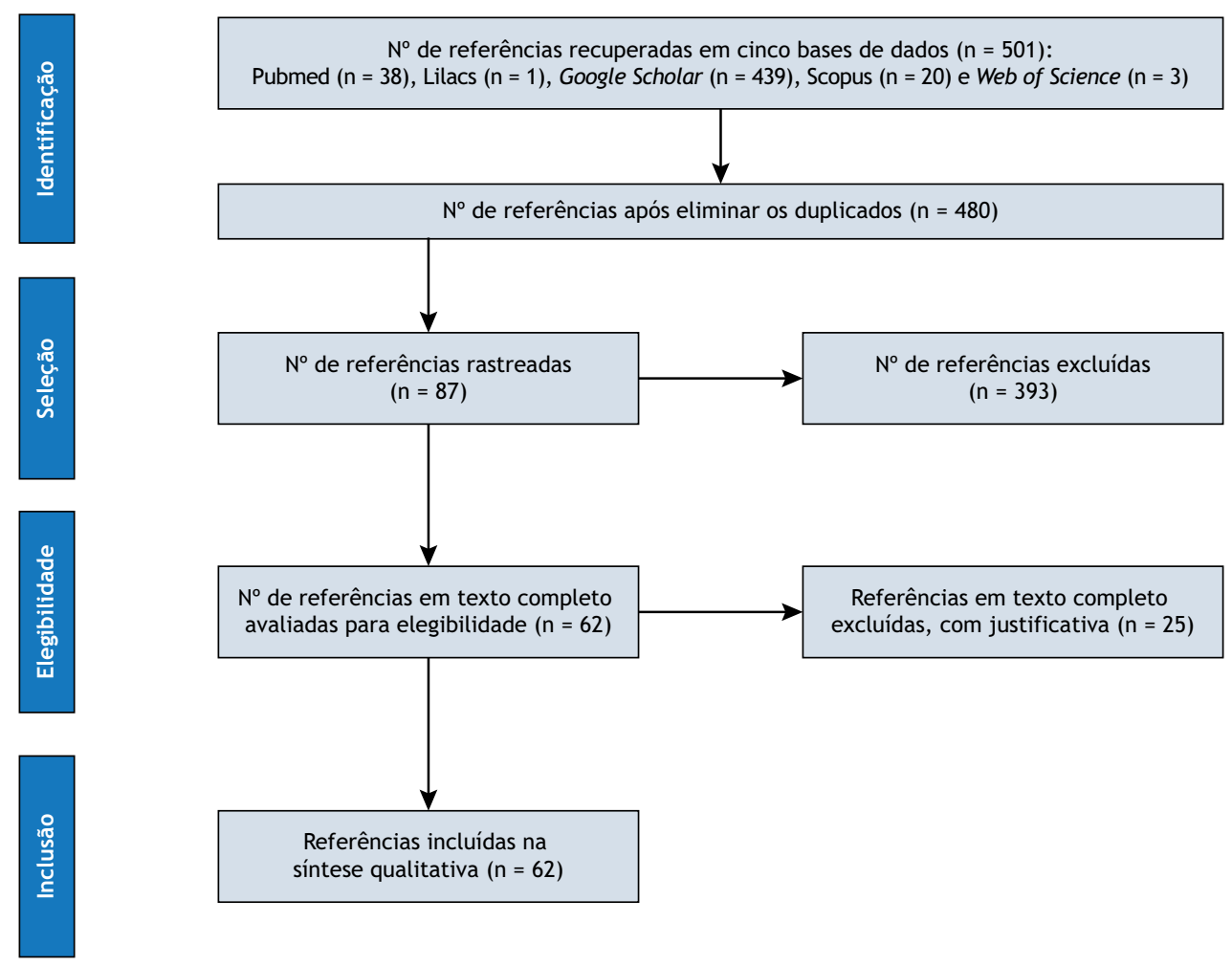

Fonte: Elaborada pelos autores, 2020.

Figura 1. Fluxograma da revisão sistemática. 
Tabela 1. Características descritivas dos estudos sobre COVID-19 incluídos com propostas One Health.

\begin{tabular}{|c|c|c|c|c|}
\hline Autores & $\begin{array}{c}\text { Tipo de } \\
\text { publicação }\end{array}$ & Periódico & $\begin{array}{l}\text { País de origem } \\
\text { dos autores }\end{array}$ & Propostas One Health \\
\hline Amirian, Levy ${ }^{11}$ & Artigo de revisão & One Health & Estados Unidos & veterinário e na agricultura. \\
\hline
\end{tabular}

\begin{tabular}{|c|c|c|c|c|}
\hline Bhatia $^{12}$ & Perspectiva & $\begin{array}{l}\text { Indian Journal of } \\
\text { Medical Research }\end{array}$ & Índia & Sistema de vigilância sensível na interface humano-animal. \\
\hline Bonilla-Aldana et al. ${ }^{13}$ & Editorial & $\begin{array}{l}\text { Advances in Animal } \\
\text { and Veterinary } \\
\text { Sciences }\end{array}$ & Colômbia e Índia & $\begin{array}{l}\text { Colaboração entre setores da saúde humana, animal e } \\
\text { ambiental nos âmbitos regional, nacional e internacional. }\end{array}$ \\
\hline Connolly ${ }^{14}$ & Artigo original & Global Policy & Escócia & $\begin{array}{l}\text { Discussão sobre a crescente urbanização e diminuição de } \\
\text { habitats animais. Alerta para a necessidade de atenção dos } \\
\text { cientistas sociais ao conceito de One Health. }\end{array}$ \\
\hline Decaro et al. ${ }^{15}$ & Artigo original & $\begin{array}{l}\text { Research in } \\
\text { Veterinary Science }\end{array}$ & $\begin{array}{l}\text { Estados Unidos e } \\
\quad \text { Itália }\end{array}$ & $\begin{array}{l}\text { Utilização da experiência da medicina veterinária no controle } \\
\text { de outros coronavírus que afetam animais para a formulação } \\
\text { de medidas contra o COVID-19. }\end{array}$ \\
\hline Dilcher et al. ${ }^{16}$ & Editorial & $\begin{array}{l}\text { New Zealand } \\
\text { Medical Journal }\end{array}$ & Nova Zelândia & $\begin{array}{l}\text { Sistemas robustos e rápidos de comunicação entre países, } \\
\text { para garantir que novos patógenos sejam rapidamente } \\
\text { identificados, caracterizados e contidos, se possível. }\end{array}$ \\
\hline Zowalaty, Järhult ${ }^{17}$ & Artigo original & One Health & $\begin{array}{c}\text { Suécia e } \\
\text { Emirados Árabes }\end{array}$ & $\begin{array}{l}\text { Vigilância de SARS-CoV-2 em hospedeiros silvestres, } \\
\text { inclusive com análise genética; conhecimento da ecologia } \\
\text { do vírus, para que situações de transmissão de alto } \\
\text { risco possam ser evitadas; medidas de ciências sociais/ } \\
\text { comportamentais para evitar interações das populações } \\
\text { humanas com os animais reservatórios; detecção de } \\
\text { hospedeiros reservatórios e remoção destes animais dos } \\
\text { mercados de animais vivos ou rigorosamente testados para } \\
\text { o vírus; redução da transmissão humano-humano, com foco } \\
\text { nas ciências sociais (mudança de comportamento para a } \\
\text { prevenção, em pessoas infectadas ou doentes); legislação } \\
\text { e medidas colaborativas entre os setores da agricultura e } \\
\text { saúde pública, com enfoque na biossegurança e na educação } \\
\text { sobre doenças zoonóticas. }\end{array}$ \\
\hline
\end{tabular}

Foddai et al. ${ }^{18} \quad$ Editorial One Health $\quad \begin{gathered}\text { Dinamarca, } \\ \text { Suécia e Itália }\end{gathered}$

Consideração sobre a experiência veterinária (legislação/ vigilância) obtida em epizootias pode oferecer conhecimentos adicionais à resposta da saúde pública ao COVID-19.

\begin{tabular}{|c|c|c|c|}
\hline Foddai et al. ${ }^{19}$ & Artigo original & One Health & \\
\hline Gollakner, Capua ${ }^{20}$ & $\begin{array}{c}\text { Comunicação } \\
\text { curta }\end{array}$ & Veterinaria Italiana & $\begin{array}{c}\text { Itália e } \\
\text { Dinamarca }\end{array}$ \\
\hline
\end{tabular}

Utilização de um protocolo de vigilância em tempo real para COVID-19 adaptado de uma metodologia veterinária.

Harmonização de diretrizes para vigilância e intervenção em espécies selvagens, em cativeiro e em animais de companhia para facilitar a compreensão da propagação viral em novas populações hospedeiras e prevenção de uma panzootia.

\begin{tabular}{|c|c|c|c|c|}
\hline Hemida, Ba Abduallah"21 & Artigo original & One Health & $\begin{array}{l}\text { Arábia Saudita e } \\
\text { Egito }\end{array}$ & $\begin{array}{l}\text { Monitoramento do vírus em hospedeiros naturais e } \\
\text { possíveis hospedeiros intermediários; descontaminação de } \\
\text { ambientes públicos; mudança de hábitos pessoais e estilo } \\
\text { de vida. }\end{array}$ \\
\hline Jacobsen $^{22}$ & Comentário & The Lancet & Estados Unidos & $\begin{array}{l}\text { Detecção de agentes etiológicos em animais de produção } \\
\text { e em animais silvestres, criando um sistema de alerta para } \\
\text { pandemias humanas. }\end{array}$ \\
\hline Kahn ${ }^{23}$ & Comentário & $\begin{array}{l}\text { International } \\
\text { Journal of } \\
\text { Epidemiology }\end{array}$ & Estados Unidos & $\begin{array}{l}\text { Proteção dos habitats da vida selvagem como as florestas } \\
\text { tropicais para evitar eventos de transbordamento de } \\
\text { patógenos zoonóticos. }\end{array}$ \\
\hline $\mathrm{Lal}^{24}$ & Editorial & $\begin{array}{l}\text { Journal of Soil and } \\
\text { Water Conservation }\end{array}$ & Estados Unidos & $\begin{array}{l}\text { Proteção do solo, água e ar. Alteração dos valores da } \\
\text { sociedade, estilo de vida e negócios promovendo o uso } \\
\text { sustentável do meio ambiente. }\end{array}$ \\
\hline Leroy et al. ${ }^{25}$ & Editorial & One Health & França & $\begin{array}{l}\text { Estudos para estabelecer o risco de transmissão de COVID-19 } \\
\text { dos humanos para os animais de estimação (zoonose reversa), } \\
\text { bem como animais infectados serem fontes de infecção para } \\
\text { os seres humanos. }\end{array}$ \\
\hline Lorusso et al. ${ }^{26}$ & Artigo original & One Health & Itália & $\begin{array}{l}\text { Maior participação de veterinários e metodologias da } \\
\text { medicina veterinária na saúde pública. }\end{array}$ \\
\hline
\end{tabular}

Continua 
Continuação

\begin{tabular}{|c|c|c|c|c|}
\hline Autores & $\begin{array}{c}\text { Tipo de } \\
\text { publicação }\end{array}$ & Periódico & $\begin{array}{l}\text { País de origem } \\
\text { dos autores }\end{array}$ & Propostas One Health \\
\hline Lorusso et al. ${ }^{27}$ & Editorial & Veterinaria Italiana & Itália & $\begin{array}{l}\text { Manter as barreiras naturais entre os reservatórios naturais de } \\
\text { vírus zoonóticos e a sociedade; classificar as doenças zoonóticas } \\
\text { em relação ao seu potencial de causar pandemias, magnitude } \\
\text { em humanos e animais e se representa um potencial agente de } \\
\text { bioterrorismo; tecnologias inovadoras de diagnóstico, inclusive } \\
\text { com sequenciamento genético de patógenos zoonóticos e } \\
\text { criação de bancos de dados mundiais; alteração na formação de } \\
\text { médicos veterinários, com aprofundamento em bioinformática, } \\
\text { genômica, ciências sociais, estatística e ecologia. }\end{array}$ \\
\hline Messmer 28 & Comentário & $\begin{array}{l}\text { Human-Wildlife } \\
\text { Interactions }\end{array}$ & Estados Unidos & $\begin{array}{l}\text { Educação em saúde realizada por parte de profissionais da } \\
\text { área One Health no sentido de promover o conhecimento } \\
\text { sobre zoonoses associadas a animais silvestres, sem instigar } \\
\text { na sociedade a ideia de que estes são pragas transmissoras de } \\
\text { doenças que devem ser eliminadas. }\end{array}$ \\
\hline Paul et al. ${ }^{29}$ & Comentário & BMJ Global Health & Bélgica e França & $\begin{array}{l}\text { As políticas globais de saúde não devem ser elaboradas como } \\
\text { modo de resposta as ameaças caso a caso, mas devem adotar } \\
\text { uma abordagem holística; política de proteção à saúde global, } \\
\text { sem fragmentações, com considerações para cada contexto; } \\
\text { políticas a longo prazo para diminuir os riscos coletivos e } \\
\text { individuais, como fatores ambientais e iniquidades sociais. }\end{array}$ \\
\hline Renda, Castro ${ }^{30}$ & Artigo original & $\begin{array}{l}\text { European Journal of } \\
\quad \text { Risk Regulation }\end{array}$ & Bélgica & Maior integração das áreas de saúde animal e saúde humana. \\
\hline Singh, Ofrin ${ }^{31}$ & Editorial & $\begin{array}{l}\text { WHO South-East Asia } \\
\text { Journal of Public } \\
\text { Health }\end{array}$ & Índia & $\begin{array}{l}\text { Sistemas de saúde verdadeiramente resilientes, com a } \\
\text { participação de setores diversos do poder público, da ciência } \\
\text { e da sociedade. }\end{array}$ \\
\hline Sun et al. ${ }^{32}$ & Artigo de revisão & $\begin{array}{l}\text { Trends in Molecular } \\
\text { Medicine }\end{array}$ & $\begin{array}{l}\text { China e Estados } \\
\text { Unidos }\end{array}$ & $\begin{array}{l}\text { Atuação interdisciplinar entre profissionais de saúde humana } \\
\text { com veterinários e zoólogos; vigilância (por diagnóstico) em } \\
\text { animais domésticos e de produção, para evitar a expansão } \\
\text { das espécies hospedeiras ou transmissão interespécies de } \\
\text { novos coronavírus para humanos. }\end{array}$ \\
\hline Thool $^{33}$ & Artigo original & $\begin{array}{l}\text { International } \\
\text { Journal of Scientific } \\
\text { Research \& } \\
\text { Engineering Trends }\end{array}$ & Índia & $\begin{array}{l}\text { Manter a barreira de espécie entre reservatórios naturais de } \\
\text { agentes patogênicos zoonóticos e a sociedade humana. }\end{array}$ \\
\hline Tilocca et al. ${ }^{34}$ & $\begin{array}{l}\text { Comunicação } \\
\text { curta }\end{array}$ & $\begin{array}{l}\text { Microbes and } \\
\text { Infection }\end{array}$ & Itália & $\begin{array}{l}\text { Investigação molecular de diferentes coronavírus em } \\
\text { comparação com o COVID-19. }\end{array}$ \\
\hline Tilocca et al. ${ }^{35}$ & Artigo original & $\begin{array}{l}\text { Microbes and } \\
\text { Infection }\end{array}$ & Itália & $\begin{array}{l}\text { Mapeamento de epítopos dos coronavírus em diversas áreas } \\
\text { geográficas, na população humana e animal, para a adoção } \\
\text { de medidas de contenção mais adequadas. }\end{array}$ \\
\hline Broucke $^{36}$ & Editorial & $\begin{array}{l}\text { Health Promotion } \\
\text { International }\end{array}$ & Bélgica & $\begin{array}{l}\text { Maior interação de pesquisadores das áreas de Promoção da } \\
\text { Saúde e de Desenvolvimento Sustentável com a iniciativa One } \\
\text { Health, as quais possuem características muito semelhantes. }\end{array}$ \\
\hline Wang et al. ${ }^{37}$ & Carta ao editor & Journal of Infection & China e Canadá & $\begin{array}{l}\text { Investigações epidemiológicas entre animais domésticos e } \\
\text { de produção; leis restritivas em relação ao mercado de vida } \\
\text { silvestre. }\end{array}$ \\
\hline Weng $^{38}$ & Artigo de Opinião & $\begin{array}{l}\text { Acta Medica } \\
\text { Mediterranea }\end{array}$ & China & $\begin{array}{l}\text { Aumentar a capacidade técnica de equipes para prevenção } \\
\text { de epidemias e pesquisa em Saúde Pública; intervenções } \\
\text { para controlar as crises psicológicas da população; garantia } \\
\text { de equipamentos de proteção individual para todos os } \\
\text { envolvidos em períodos de epidemia; vigilância, prevenção e } \\
\text { controle no "período fraco" da epidemia. }\end{array}$ \\
\hline
\end{tabular}

Fonte: Elaborada pelos autores, 2020.

(Tabelas 1 e 2 ). Apenas uma publicação $(1,6 \%)$ era na língua portuguesa e as demais em inglês. Uma publicação em italiano foi encontrada durante as buscas nas bases de dados, porém foi excluída por não se enquadrar nos critérios de inclusão. Dentre estas publicações, 28 (45,2\%) apresentavam propostas para a vigilância em saúde da COVID-19 por meio de abordagem One Health, com grande variedade de propostas. As investigações relacionadas ao vírus, aos hospedeiros e à biotecnologia foram as propostas mais frequentes $(19 ; 67,9 \%)$.
A participação de médicos veterinários em estratégias One Health para o controle de COVID-19, utilizando os protocolos de vigilância e experiências acumuladas nesta área, também foi frequente $(6 ; 21,4 \%)$ (Tabela 1). Outras 34 (54,8\%) publicações apenas relatavam a importância dessa abordagem no combate ao vírus (Tabela 2 ).

No período avaliado, foi observado um incremento no número de publicações ao longo dos meses do ano de 2020 (Figura 2). 
Tabela 2. Características dos estudos sobre COVID-19 incluídos com relatos da importância da abordagem One Health.

\begin{tabular}{|c|c|c|c|}
\hline Autores & Tipo de publicação & Periódico & País de origem dos autores \\
\hline Ahmad, Hui ${ }^{39}$ & Carta ao editor & Human Vaccines \& Immunotherapeutics & China \\
\hline Burkle 40 & Relato especial & Prehospital and Disaster Medicine & Estados Unidos \\
\hline Chatterjee et al. ${ }^{41}$ & Artigo de revisão & Indian Journal of Medical Research & Índia \\
\hline Chaves, Bellei ${ }^{42}$ & Editorial & Revista de Medicina & Brasil \\
\hline Chen et al. ${ }^{43}$ & Carta ao editor & Journal of Infection & China \\
\hline Dhama et al. ${ }^{44}$ & Artigo de revisão & Preprints* & Índia, Nepal, Colômbia e Polônia \\
\hline Fasina ${ }^{45}$ & Perspectiva & Asian Pacific Journal of Tropical Medicine & África do Sul \\
\hline Helmy et al. ${ }^{46}$ & Artigo de revisão & Journal of Clinical Medicine & Egito, Estados Unidos e Alemanha \\
\hline Jørgensen, Neves ${ }^{47}$ & Carta ao editor & Tidsskrift for Den norskelegeforening & Noruega \\
\hline Kapata et al. ${ }^{48}$ & Editorial & $\begin{array}{c}\text { International Journal of Infectious } \\
\text { Diseases }\end{array}$ & $\begin{array}{c}\text { Zâmbia, Nigéria, Congo, Alemanha, } \\
\text { Etiópia e Inglaterra }\end{array}$ \\
\hline Kilic et al. ${ }^{49}$ & Opinião & Northern Clinics of Istanbul & Turquia \\
\hline Kock et al. ${ }^{50}$ & Comentário & Lancet Planet Health & $\begin{array}{c}\text { Estados Unidos, Alemanha, Tanzânia, } \\
\text { Vietnã e Inglaterra }\end{array}$ \\
\hline Lum, Tambyah ${ }^{51}$ & Editorial & Singapore Medical Journal & Singapura \\
\hline Mackenzie, Willians ${ }^{52}$ & Editorial & Microbiology Australia & Austrália \\
\hline Malik et al. ${ }^{53}$ & Artigo original & Preprints ${ }^{*}$ & Índia, Japão, Colômbia \\
\hline Malik et al. ${ }^{54}$ & Artigo de revisão & Preprints* & Índia, Nepal e Colômbia \\
\hline Malik et al. ${ }^{55}$ & Comunicação curta & Veterinary Quarterly & Índia, Irã e Tailândia \\
\hline Marty, Jones ${ }^{56}$ & Editorial & One Health & Austrália \\
\hline Murdoch, French ${ }^{57}$ & Editorial & New Zealand Medical Journal & Nova Zelândia \\
\hline Musa et al. ${ }^{58}$ & Carta ao editor & $\begin{array}{c}\text { The Journal of Infection in Developing } \\
\text { Countries }\end{array}$ & China, Paquistão e Sudão \\
\hline Parry ${ }^{59}$ & Carta ao editor & Forensic Science International: Reports & Estados Unidos \\
\hline Qian et al. ${ }^{60}$ & Comentário & Infectious Diseases of Poverty & China \\
\hline Ravikumar et al. ${ }^{61}$ & Artigo original & $\begin{array}{c}\text { International Journal of Recent Scientific } \\
\text { Research }\end{array}$ & Índia \\
\hline Rodriguez-Morales et al. ${ }^{6}$ & Editorial & Le Infezioni in Medicina & $\begin{array}{c}\text { Colômbia, Peru, Estados Unidos, Itália e } \\
\text { Venezuela }\end{array}$ \\
\hline Rodriguez-Morales et al. ${ }^{62}$ & Artigo de revisão & Journal of Pure and Applied Microbiology & Colômbia, Índia e Nepal \\
\hline Stoffel et al. ${ }^{63}$ & Carta ao editor & Transboundary and Emerging Diseases & Suíça \\
\hline Trilla9 & Editorial & Medicina Clínica & Espanha \\
\hline Vicente $^{64}$ & Carta ao editor & $\begin{array}{l}\text { Revista da Sociedade Brasileira de } \\
\text { Medicina Tropical }\end{array}$ & Brasil \\
\hline Wilson, Chen ${ }^{65}$ & Editorial & Journal of Travel Medicine & Estados Unidos \\
\hline Xiong et al. ${ }^{66}$ & Artigo original & BioRxiv* & China e Canadá \\
\hline Yadav et al. ${ }^{67}$ & Artigo original & Indian Journal of Medical Research & Índia \\
\hline Yassine, Shah ${ }^{68}$ & Entrevista & Expert Review of Anti-infective Therapy & Catar \\
\hline Zumla, Niederman ${ }^{69}$ & Editorial & Current Opinion in Pulmonary Medicine & Estados Unidos e Inglaterra \\
\hline
\end{tabular}

Fonte: Elaborada pelos autores, 2020.

*Repositório de preprints.

\section{DISCUSSÃO}

No período avaliado foi observado um aumento no número de publicações científicas, prioritariamente as publicações de opinião e de formato curto. Os artigos originais que apresentavam propostas concretas foram a minoria. Também foi observada uma abrangência nas temáticas apresentadas, estando essas, em acordo com as premissas da One Health.

A abordagem One Health tem sido amplamente discutida nas últimas décadas e surge da necessidade de dar uma resposta interdisciplinar aos problemas que afetam a saúde humana, dos animais e do ambiente ${ }^{70}$. A aplicabilidade do conceito (One Health) foi motivada pelas rápidas mudanças ocorridas nas últimas décadas na densidade demográfica da população, urbanização, expansão da população (aliadas à invasão de ecossistemas), intensificação das práticas extrativistas e do consumo de alimentos e das fontes de energia (renováveis ou não) do planeta ${ }^{71}$.

A expressão One Health foi adotada inicialmente com grande entusiasmo pelos médicos veterinários e pelas agências 


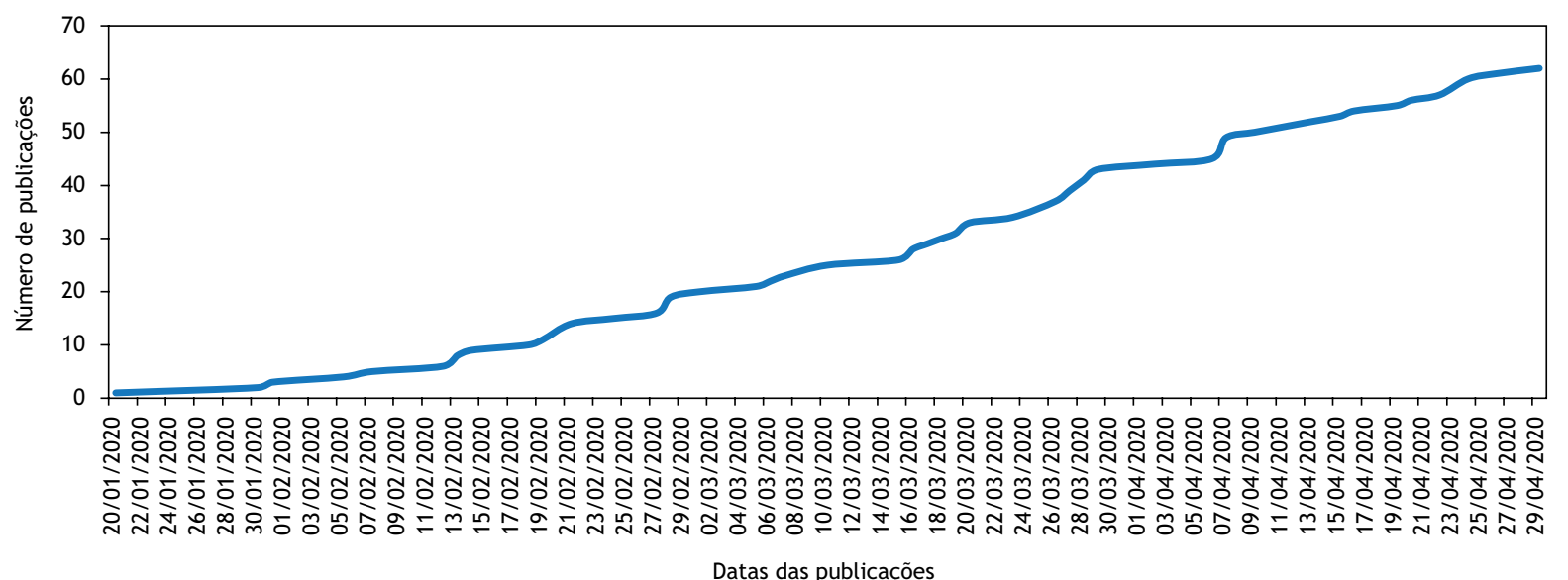

Fonte: Elaborada pelos autores, 2020.

Figura 2. Número de publicações com a abordagem One Health para COVID-19 segundo os critérios de busca da revisão sistemática, de acordo com a data da sua publicação.

internacionais encarregadas do controle de zoonoses, principalmente a Organização para Agricultura e Alimentação (FAO), Organização Mundial de Saúde (OMS) e Organização Mundial de Saúde Animal (OIE). Em todo o mundo, esse grupo de profissionais tem promovido o conceito de One Health para tratar de questões como segurança alimentar, resistência antimicrobiana, mudança climática e vínculo humano-animal ${ }^{72}$.

A emergência de vírus zoonóticos com o potencial de causar doenças pandêmicas, incluindo extensa mortalidade humana, criou várias crises internacionais ${ }^{73}$. Nesse sentido, governos e cientistas em todo o mundo reconheceram a necessidade de uma maior colaboração multiprofissional para prevenir e controlar as zoonoses, e que essa colaboração deveria incluir não apenas os médicos e os veterinários, mas também biólogos, especialistas em vida selvagem, ambientalistas, antropólogos, economistas, sociólogos entre outros ${ }^{71}$.

Estima-se que $60 \%$ dos patógenos humanos emergentes são de origem zoonótica. Desses, mais de $71 \%$ têm origem em animais silvestres. Esses patógenos podem mudar de hospedeiro adquirindo novas combinações genéticas que têm potencial patogênico alterado, por mudanças de comportamento ou por características socioeconômicas, ambientais ou ecológicas dos hospedeiros ${ }^{74}$.

Na pandemia da COVID-19, observou-se que o consumo de animais silvestres como fonte proteica pode ter sido o responsável pelo escape zoonótico desse patógeno na China ${ }^{75}$. Muitas doenças zoonóticas estão associadas ao consumo de carne de animais silvestres. A carne de caça é considerada uma iguaria em muitos países e este aspecto tem motivado o crescimento dessa nova modalidade comercial $^{74}$. 0 rastreamento, captura, manuseio, abate no campo e transporte de carcaças envolvem riscos de transmissão de patógenos entre espécies ${ }^{74,76}$. Além disso, a produção e a comercialização de animais silvestres em criatórios exigem uma fiscalização de toda a cadeia produtiva até a ingestão da carne abatida, fato este que não é rigorosamente seguido em muitos países ${ }^{77}$.

No contexto da pandemia da COVID-19, as abordagens em One Health têm proposto o fortalecimento dessas colaborações entre os setores da saúde humana, animal e ambiental nos âmbitos regional, nacional e internacional ${ }^{13}$. Idealmente, as abordagens deverão utilizar-se das experiências das diversas classes profissionais para o enfrentamento da pandemia.

A integração das ações da One Health vai além da atuação profissional local, mas essa deve ser globalizada, buscando o desenvolvimento de sistemas de comunicação mais robustos e interligados, o que possibilitaria a adoção de estratégia de contenção de patógenos emergentes, antes da sua introdução e do seu estabelecimento ${ }^{16}$.

A busca por estratégias para melhorar as formas de comunicação com a população deverá ser discutida no contexto da Saúde Única ${ }^{78}$. Dessa forma, o desenvolvimento de ações de educação e promoção da saúde realizada por profissionais da One Health pode promover o conhecimento sobre zoonoses associadas a animais silvestres, sem instigar na sociedade a ideia de que estes são pragas transmissoras de doenças que devem ser eliminadas ${ }^{28}$.

Provavelmente, os mercados de animais vivos desempenham um papel importante nesse processo e precisam ser abordados ${ }^{17}$. No entanto, é crucial considerar o contexto cultural desses mercados, o que significa que novamente as ciências sociais são importantes nesse processo ${ }^{78}$. Além disso, isso significa que a solução mais viável pode não ser fechar os mercados de animais vivos, mas talvez "setorizá-los" para que menos espécies diferentes se misturem em um mercado específico e que os hospedeiros intermediários específicos para SARS-CoV-2 possam ser removidos dos mercados ou rigorosamente testados para o vírus ${ }^{79}$.

Entre as práticas prevalentes da One Health recomendadas nessa revisão, observamos a necessidade de adoção de investigações ecoepidemiológicas que possibilitem ampliar o conhecimento sobre a ecologia do vírus, seus reservatórios e hospedeiros com a finalidade da proposição de medidas de prevenção e controle ${ }^{17}$. A manutenção de barreiras entre os reservatórios naturais de vírus zoonóticos e a sociedade também é proposta ${ }^{27}$. 
Também foi vista como necessária a inserção do profissional da One Health como gestor dos sistemas de Vigilância Epidemiológica e da Atenção Primária ${ }^{29}$. A vigilância de patógenos emergentes, para ser efetiva, deve ser uma ação continuada programática e não pode ser realizada pontualmente frente a ocorrência de surtos epidêmicos ${ }^{80}$.

Ademais, os profissionais que atuam em One Health devem ter em mãos sistemas de informação em saúde robustos e interligados entre os diferentes segmentos, que possibilitem o monitoramento das tendências e a sinalização preditiva de situações de urgência, que vão além da emergência do patógeno, mais também das capacidades locais de assistência a população ${ }^{81,82}$.

Em momentos de crise, cabe ao profissional da One Health identificar a necessidade de intervenções para controlar as situações de ordem psicológica da população. Em uma pandemia, as pessoas ficam mais vulneráveis, por isso a identificação destes cenários e a proposição de estratégias que minimizem o estresse gerado pela pandemia são de extrema importância ${ }^{83}$.

\section{CONCLUSÕES}

Com o conjunto de artigos levantados na revisão sistemática foi possível avaliar que as abordagens de One Health são tão amplas quanto o seu conceito, e que o contexto pandêmico da COVID-19 pode ser um importante marco para o estabelecimento desse enfoque multidisciplinar que tem sido promovido nas últimas décadas, como a solução mais ampla e apropriada para os problemas de saúde.
O desafio da One Health converge para a prática multiprofissional, aproveitando os diferentes saberes, para solução de problemas que são multicausais. Nesse sentido, a compreensão mais ampla sobre os determinantes de saúde-adoecimento-cuidado deve ser retomada e discutida de forma colaborativa.

Ainda sobre o conjunto de informações consolidadas, fica evidente que as questões biológicas (investigações relacionadas ao vírus, aos hospedeiros e à biotecnologia) foram os temas mais discutidos como estratégias de One Health, em detrimento das questões sociais, econômicas e comportamentais. Tal observação evidencia a necessidade da adesão de outras classes profissionais (sociólogos, economistas e psicólogos) como protagonistas da aplicação dos conceitos da One Health.

Não menos importante, observa-se a necessidade de políticas governamentais, que devem ser adotadas para garantir as ações multiprofissionais de saúde. Igualmente, os conselhos profissionais devem estimular e balizar as atuações colaborativas, buscando dar o amparo legal das atuações de One Health dentro de cada profissão.

Com a abrangência de propostas que foram aqui discutidas, torna-se imperiosa a aplicabilidade da One Health no contexto pandêmico vivenciado pela COVID-19. A saúde humana, animal e a proteção do solo, água, ar e meio ambiente em geral são indissociáveis. Cabe aos profissionais a promoção e a aplicação deste conceito, de forma articulada e interdisciplinar, buscando promover o desenvolvimento da sociedade de forma sustentável.

\section{REFERÊNCIAS}

1. Cui J, Li F, Shi-Li Z. Origin and evolution of pathogenic coronaviruses. Nat Rev Microbiol. 2019;17(3):181-92. https://doi.org/10.1038/s41579-018-0118-9

2. Millan-Oñate J, Rodríguez-Morales AJ, Camacho-Moreno G, Mendoza-Ramírez $\mathrm{H}$, Rodríguez-Sabogal IA, Álvarez-Moreno C. A new emerging zoonotic virus of concern: the 2019 novel coronavirus (SARS CoV-2). Infect. 2020;24(3):187-92. https://doi.org/10.22354/in.v24i3.848

3. Mattar S, González M. Zoonotic emergence of coronavirus: a potential public risk for Latin America. Rev MVZ Cordoba. 2018;23(3):6775-7. https://doi.org/10.21897/rmvz.1408

4. Lipsitch $M$, Swerdlow DL, Finelli L. Defining the epidemiology of COVID-19: studies needed. N Engl J Med. 2020;382:1194-6. https://doi.org/10.1056/NEJMp2002125

5. Zhu N, Zhang D, Wang W, Li X, Yang B, Song J et al. A novel coronavirus from patients with pneumonia in China, 2019. N Engl J Med. 2020;382:727-33. https://doi.org/10.1056/NEJMoa2001017

6. Rodriguez-Morales AJ, Bonilla-Aldana DK, Balbin-Ramon GJ, Rabaan AA, Sah R, Paniz-Mondolfi A et al. History is repeating itself: a probable zoonotic spillover as a cause of an epidemic: the case of 2019 novel coronavirus. Infez Med. 2020;28(1):3-5.

7. Gorbalenya AE, Snijder EJ, Spaan WJ. Severe acute respiratory syndrome coronavirus phylogeny: toward consensus. J Virol. 2004;78(15):7863-66. https://doi.org/10.1128/JVI.78.15.7863-7866.2004

8. Chu DKW, Poon LLM, Gomaa MM, Shehata MM, Perera RAPM, Zeid DA et al. Coronaviruses in dromedary camels. Egypt Emerg Infect Dis. 2014;20(6):1049-53. https://doi.org/10.3201/eid2006.140299

9. Trilla A. One world, one health: the novel coronavirus COVID-19 epidemic. MedClin (Barc). 2020;154(5):175-7. https://doi.org/10.1016/j.medcle.2020.02.001

10. Galvão TF, Pansani TSA, Harrad D, tradutores. Principais itens para relatar revisões sistemáticas e meta-análises: a recomendação Prisma. Epidemiol Serv Saude. 2015;24(2):335-42. https://doi.org/10.5123/S1679-49742015000200017

11. Amirian ES, Levy JK. Current knowledge about the antivirals remdesivir (GS-5734) and GS-441524 as therapeutic options for coronaviruses. One Health. 2020;9:1-5. https://doi.org/10.1016/j.onehlt.2020.100128

12. Bhatia R. Need for integrated surveillance at humananimal interface for rapid detection \& response to emerging coronavirus infections using one health approach. Indian J Med Res. 2020;151(2-3):132-5. https://doi.org/10.4103/ijmr.IJMR_623_20 
13. Bonilla-Aldana DK, Dhama K, Rodriguez-Morales AJ. Revisiting the one health approach in the context of COVID-19: a look into the ecology of this emerging disease. Adv Anim Vet Sci. 2020;8(3):234-7. https://doi.org/10.17582/journal.aavs/2020/8.3.234.237

14. Connolly J. Global crisis leadership for disease-induced threats: one health and urbanisation: 'one health' and urbanisation. Glob Policy. 2020;11(3):283-92. https://doi.org/10.1111/1758-5899.12806

15. Decaro N, Martella V, Saif LJ, Buonavoglia C. COVID-19 from veterinary medicine and one health perspectives: what animal coronaviruses have taught us. Res Vet Sci. 2020;131:21-3. https://doi.org/10.1016/j.rvsc.2020.04.009

16. Dilcher M, Werno A, Jennings LC. SARS-CoV-2: a novel deadly virus in a globalised world. N Z Med J. 2020;133(1510):6-11.

17. Zowalaty ME, Järhult JD. From SARS to COVID-19: a previously unknown SARS-related coronavirus (SARS CoV-2) of pandemic potential infecting humans: call for a one health approach. One Health. 2020;9:1-6. https://doi.org/10.1016/j.onehlt.2020.100124

18. Foddai A, Lindberg A, Lubroth J, Ellis-Iversen J. Surveillance to improve evidence for community control decisions during the COVID-19 pandemic: opening the animal epidemic toolbox for public health. One Health. 2020;9:1-2. https://doi.org/10.1016/j.onehlt.2020.100130

19. Foddai A, Lubroth J, Ellis-Iversen J. Base protocol for real time active random surveillance of coronavirus disease (COVID-19): adapting veterinary methodology to public health. One Health. 2020;9:1-5. https://doi.org/10.1016/j.onehlt.2020.100129

20. Gollakner R, Capua I. Is COVID-19 the first pandemic that evolves into a panzootic? Vet Ital. 2020:56(1):7-8. https://doi.org/10.12834/Vetlt.2246.12523.1

21. Hemida MG, Ba Abduallah MMB. The SARS-CoV-2 outbreak from a one health perspective. One Health. 2020:1-24. https://doi.org/10.1016/j.onehlt.2020.100127

22. Jacobsen KH. Will COVID-19 generate global preparedness? The Lancet. 2020;395(10229):1013-4. https: //doi.org/10.1016/S0140-6736(20)30559-6

23. Kahn LH. Commentary on: the SARS, MERS and novel coronavirus (COVID-19) epidemics, the newest and biggest global health threats: what lessons have we learned? A one health approach to coronaviruses. Int J Epidemiol. 2020:13. https://doi.org/10.1093/ije/dyaa071

24. Lal R. Soil science beyond COVID-19. J Soil Water Conserv. 2020:1-3. https://doi.org/10.2489/jswc.2020.0408A

25. Leroy EM, Gouilh MA, Brugère-Picoux J. The risk of SARSCoV-2 transmission to pets and other wild and domestic animals strongly mandates a one-health strategy to control the COVID-19 pandemic. One Health. 2020:1-4. https://doi.org/10.1016/j.onehlt.2020.100133

26. Lorusso A, Calistri P, Petrini A, Savini G, Decaro N. Novel coronavirus (SARS-CoV-2) epidemic: a veterinary perspective. Vet Ital. 2020;56(1):5-10. https://doi.org/10.12834/Vetlt.2173.11599.1
27. Lorusso A, Calistri P, Mercante MT, Monaco F, Portanti O, Marcacci M et al. A one-health approach for diagnosis and molecular characterization of SARS-CoV-2 in Italy. One Health. 2020;10:1-6. https://doi.org/10.1016/j.onehlt.2020.100135

28. Messmer TA. Humans, wildlife, and our environment: one health is the common link. Hum Wildl Interact. 2020;14(1):137-40. https://doi.org/10.26077/yasd-7r53

29. Paul E, Brown GW, Ridde V. COVID-19: time for paradigm shift in the nexus between local, national and global health. BMJ Glob Health. 2020;5(4):1-5. https://doi.org/10.1136/bmjgh-2020-002622

30. Renda A, Castro R. Towards stronger EU governance of health threats after the COVID-19 pandemic. Eur J Risk Regul. 2020:1-10. https://doi.org/10.1017/err.2020.34

31. Singh PK, Ofrin RH. Quo vadis after COVID-19: a new path for global emergency preparedness? WHO South-East Asia J Public Health. 2020;9(1):1-4. https://doi.org/10.4103/2224-3151.282988

32. Sun J, He WT, Wang L, Lai A, Ji X, Zhai X et al. COVID19: epidemiology, evolution, and cross-disciplinary perspectives. Trends Mol Med. 2020;26(5):483-95. https://doi.org/10.1016/j.molmed.2020.02.008

33. Thool A. Temperature dependence and survivorship of COVID-19: myth or fact. Int J Sci Res. 2020;6(2):730-3.

34. Tilocca B, Soggiu A, Musella V, Britti D, Sanguinetti M, Urbani A et al. Molecular basis of COVID-19 relationships in different species: a one health perspective. Microbes Infect. 2020:1-3. https://doi.org/10.1016/j.micinf.2020.03.002

35. Tilocca B, Soggiu A, Sanguinetti M, Musella V, Britti D, Bonizzi L et al. Comparative computational analysis of SARS-CoV-2 nucleocapsid protein epitopes in taxonomically related coronaviruses. Microbes Infect. 2020:1-7. https://doi.org/10.1016/j.micinf.2020.04.002

36. Broucke $S$. Why health promotion matters to the COVID-19 pandemic, and vice versa. Health Promot Int. 2020:1-6. https://doi.org/10.1093/heapro/daaa042

37. Wang R, Zhang X, Irwin DM, Shen Y. Emergence of SARS-like coronavirus poses new challenge in China. J Infect. 2020;80(3):350-71. https://doi.org/10.1016/j.jinf.2020.01.017

38. Weng Y. Analysis of prevention and control strategy of COVID-19 epidemic in China based on the concept of one health: an opinion paper. Acta Medica Mediterr. 2020;36:1167-74. https://doi.org/10.19193/0393-6384_2020_2_183

39. Ahmad T, Hui J. One health approach and coronavirus disease 2019. Hum Vaccines Immunother. 2020;16(4):931-2. https://doi.org/10.1080/21645515.2020.1732168

40. Burkle FM. Political intrusions into the international health regulations treaty and its impact on management of rapidly emerging zoonotic pandemics: what history tells us. Prehospital Disaster Med. 2020:1-5. https://doi.org/10.1017/S1049023X20000515 
41. Chatterjee P, Nagi N, Agarwal A, Das B, Banerjee S, Sarkar S et al. The 2019 novel coronavirus disease (COVID-19) pandemic: a review of the current evidence. Indian J Med Res. 2020;151(2-3):147-59. https://doi.org/10.4103/ijmr.IJMR_519_20

42. Chaves TSS, Bellei N. SARS-CoV-2, o novo coronavírus: uma reflexão sobre a saúde única (one health) e a importância da medicina de viagem na emergência de novos patógenos. Rev Med. 2020;99(1):1-4. https://doi.org/10.11606/issn.1679-9836.v99i1pi-iv

43. Chen L, Cai J, Lin Q, Xiang B, Ren T. Imported COVID-19 cases pose new challenges for China. J Infect. 2020:1-7. https://doi.org/10.1016/j.jinf.2020.03.048

44. Dhama K, Patel SK, Sharun K, Pathak M, Tiwari R, Yatoo Ml et al. SARS-CoV-2: jumping the species barrier, lessons from SARS and MERS, its zoonotic spillover, transmission to humans, preventive and control measures and recent developments to counter this pandemic virus. Preprints. 2020:1-38. https://doi.org/10.20944/preprints202004.0011.v1

45. Fasina FO. Novel coronavirus (2019-nCoV) update: what we know and what is unknown. Asian Pac J Trop Med. 2020;13(3):97-8. https://doi.org/10.4103/1995-7645.277795

46. Helmy YA, Fawzy M, Elaswad A, Sobieh A, Kenney SP, Shehata AA. The COVID-19 pandemic: a comprehensive review of taxonomy, genetics, epidemiology, diagnosis, treatment, and control. J Clin Med. 2020;9(4):1-29. https://doi.org/10.3390/jcm9041225

47. Jørgensen HJ, Neves C. COVID-19: one world, one health. Tidsskr Nor Laegeforen. 2020;140(5):1-3. https://doi.org/10.4045/tidsskr.20.0212

48. Kapata N, Ihekweazu C, Ntoumi F, Raji T, Chanda-Kapata P, Mwaba P et al. Is Africa prepared for tackling the COVID-19 (SARS-CoV-2) epidemic: lessons from past outbreaks, ongoing pan-african public health efforts, and implications for the future. Int J Infect Dis. 2020;93:233-6. https://doi.org/10.1016/j.ijid.2020.02.049

49. Kilic AU, Kara F, Alp E, Doganay M. New threat: 2019 novel coronavirus infection and infection control perspective in Turkey. North Clin Istanb. 2020;7(2):95-8. https://doi.org/10.14744/nci.2020.38159

50. Kock RA, Karesh WB, Veas F, Velavan TP, Simons D, Mboera LEG et al. 2019-nCoV in context: lessons learned? Lancet Plan Health. 2020;4(3):e87-8. https://doi.org/10.1016/S2542-5196(20)30035-8

51. Lum L, Tambyah P. Outbreak of COVID-19: an urgent need for good science to silence our fears? Singapore Med J. 2020;61(2):55-7. https://doi.org/10.11622/smedj.2020018

52. Mackenzie JS, Willians D. Zoonoses. Microbiol Aust. 2020;41(1);3-5. https://doi.org/10.1071/MA20002

53. Malik YS, Kumar N, Sircar S, Kaushik R, Bhatt S, Dhama $\mathrm{K}$ et al. Pandemic coronavirus disease (COVID-19): challenges and a global perspective. Prepints. 2020:1-40. https://doi.org/10.20944/preprints202004.0469.v1
54. Malik YS, Sircar S, Bhat S, Vinodhkumar OR, Tiwari R, Sah R et al. Emerging coronavirus disease (COVID-19), a pandemic public health emergency with animal linkages: current status update. Preprints. 2020:1-40. https://doi.org/10.20944/preprints202003.0343.v1

55. Malik YS, Sircar S, Bhat S, Sharun K, Dhama K, Dadar M et al. Emerging novel coronavirus (2019-nCoV): current scenario, evolutionary perspective based on genome analysis and recent developments. Vet Q. 2020;40(1):68-76. https://doi.org/10.1080/01652176.2020.1727993

56. Marty AM, Jones MK. The novel coronavirus (SARS(oV-2) is a one health issue. One Health. 2020;9:1-2. https://doi.org/10.1016/j.onehlt.2020.100123

57. Murdoch DR, French NP. COVID-19: another infectious disease emerging at the animal-human interface. $\mathrm{N} Z$ Med J. 2020;133(1510):12-5.

58. Musa TH, Ahmad T, Khan M, Haroon H, Wei P. Global outbreak of 2019-nCoV: a new challenge? J Infect Dev Ctries. 2020;14(3):244-5. https://doi.org/10.3855/jidc.12530

59. Parry NMA. COVID-19 and pets: when pandemic meets panic. Forensic Sci Int Rep. 2020;2:1-4. https://doi.org/10.1016/j.fsir.2020.100090

60. Qian X, Ren R, Wang Y, Guo Y, Fang J, Wu ZD et al. Fighting against the common enemy of COVID-19: a practice of building a community with a shared future for mankind. Infect Dis Poverty. 2020;9(1):1-6. https://doi.org/10.1186/s40249-020-00650-1

61. Ravikumar TS, Rajeev A, Limalemla J, Desham C. Urgent need for revamping competencies among medical professionals in the times of one-health: lessons from the COVID-19 death rates. Int J Recent Sci Res. 2020;11(3):37927-9. https://doi.org/10.24327/IJRSR

62. Rodriguez-Morales AJ, Bonilla-Aldana DK, Tiwari R, Sah R, Rabaan AA, Dhama K. COVID-19, an emerging coronavirus infection: current scenario and recent developments: an overview. J Pure Appl Microbiol. 2020;14(1):5-12. https://doi.org/10.22207/JPAM.14.1.02

63. Stoffel C, Schuppers M, Buholzer P, Muñoz V, Lechner I, Sperling $\mathrm{U}$ et al. The ongoing crises in China illustrate that the assessment of epidemics in isolation is no longer sufficient. Transbound Emerg Dis. 2020:67(3):1043-4. https://doi.org/10.1111/tbed.13536

64. Vicente $C R$. Is Brazil prepared for the new era of infectious disease epidemics? Rev Soc Bras Med Trop. 2020;53:1-3. https://doi.org/10.1590/0037-8682-0218-2020

65. Wilson ME, Chen LH. Travellers give wings to novel coronavirus (2019-nCoV). J Travel Med. 2020;27(2):1-3. https://doi.org/10.1093/jtm/taaa015

66. Xiong $\mathrm{C}$, Jiang $\mathrm{L}$, Chen $\mathrm{Y}$, Jiang Q. Evolution and variation of 2019-novel coronavirus. BioRxiv. 2020:1-17. https://doi.org/10.1101/2020.01.30.926477

67. Yadav P, Shete-Aich A, Nyayanit DA, Pardeshi P, Majumdar $\mathrm{T}$, Balasubramanian $\mathrm{R}$ et al. Detection of coronaviruses in Pteropus \& Rousettus species of bats from different states of India. Indian J Med Res. 2020;151(2-3):226-35. https://doi.org/10.4103/ijmr.IJMR_795_20 
68. Yassine HM, Shah Z. How could artificial intelligence aid in the fight against coronavirus? Expert Rev Anti Infect Ther. 2020;18(6):493-7. https: / / doi.org/10.1080/14787210.2020.1744275

69. Zumla A, Niederman MS. The explosive epidemic outbreak of novel coronavirus disease 2019 (COVID-19) and the persistent threat of respiratory tract infectious diseases to global health security. Curr Opin Pulm Med. 2020;26(3):193-6. https://doi.org/10.1097/MCP.0000000000000676

70. Zinsstag J, Schelling E, Waltner-Toews D, Tanner M. From one medicine to one health and systemic approaches to health and well-being. Prev Vet Med. 2020;101(3-4):148-56. https://doi.org/10.1016/j.prevetmed.2010.07.003

71. Gibbs EPJ. The evolution of one health: a decade of progress and challenges for the future. Vet Rec. 2014;174(4):85-91. https://doi.org/10.1136/vr.g143

72. Lee K, Brumme ZL. Operationalizing the one health approach: the global governance challenges. Health Policy Plan. 2013;28(7):778-85. https: / /doi.org/10.1093/heapol/czs127

73. Gibbs EP. Emerging zoonotic epidemics in the interconnected global community. Vet Rec. 2005;157(22):673-9. https://doi.org/10.1136/vr.157.22.673

74. Cutler SJ, Fooks AR, Poel WH. Public health threat of new, reemerging, and neglected zoonoses in the industrialized world. Emerg Infect Dis. 2010;16(1):1-7. https://doi.org/10.3201/eid1601.081467

75. Zhang T, Wu Q, Zhang Z. Probable pangolin origin of SARS-CoV-2 associated with the COVID-19 outbreak. Curr Biol. 2020;30(7):1346-51. https://doi.org/10.1016/j.cub.2020.03.022

76. Carrijo LMP, Limongi JE. Zoonoses relacionadas ao trabalho: riscos biológicos associados ao manejo da vida silvestre no bioma cerrado. Sci Plena. 2019;15(10):5-12. https://doi.org/10.14808/sci.plena.2019.107501

77. Wolfe ND, Daszak P, Kilpatrick AM, Burke DS. Bushmeat hunting, deforestation, and prediction of zoonotic disease emergence. Emerg Infect Dis. 2005;11(12):1822-7. https://doi.org/10.3201/eid1112.040789

78. Oliveira SV, Limongi JE. Conhecimentos, atitudes e práticas em saúde: um método epidemiológico preliminar nas abordagens de comunicação em saúde. J Health NPEPS. 2020;5(1):14-9. https://doi.org/10.30681/252610104530

79. Lu J, Milinovich GJ, Hu W. A brief historical overview of emerging infectious disease response in China and the need for a one health approach in future responses. One Health. 2016;2:99-102. https://doi.org/10.1016/j.onehlt.2016.07.001

80. Oliveira SV, Caldas EP, Limongi JE, Gazeta GS. Avaliação dos conhecimentos e atitudes de prevenção sobre a febre maculosa entre profissionais de saúde no Brasil. J Health Biol Sci. 2016:4(3):152-9. https://doi. org/10.12662/2317-3076jhbs.v4i3.851.p152-159.2016

81. Dias FLT, Mendonça FD, Pinto GM, Borges ISC, Oliveira SV. Doenças respiratórias no triângulo mineiro: análise epidemiológica e projetiva com a pandemia de COVID-19. J Health Biol Sci. 2020;8(1):1-6. https://doi.org/10.12662/2317-3076jhbs. v8i1.3219.p1-6.2020

82. Mendonça FD, Rocha SS, Pinheiro DLP, Oliveira SV. Região norte do Brasil e a pandemia de COVID-19: análise socioeconômica e epidemiológica. J Health NPEPS. 2020;5(1):20-37. https://doi.org/10.30681/252610104535

83. Lima CA, Alves PMR, Oliveira CJB, Oliveira TRN, Barbosa KB, Marcene $\mathrm{HC}$ et al. Letter to the editor: COVID-19: isolations, quarantines and domestic violence in rural areas. SciMed J. 2020;2(1):44-5. https://doi.org/10.28991/SciMedJ-2020-0201-7

\section{Contribuição dos Autores}

Limongi JE - Concepção, planejamento (desenho do estudo), aquisição, análise, interpretação dos dados e redação do trabalho. Oliveira SV - Planejamento (desenho do estudo), aquisição, análise, interpretação dos dados e redação do trabalho. Os autores aprovaram a versão final do trabalho.

Conflito de Interesse

Os autores informam não haver qualquer potencial conflito de interesse com pares e instituições, políticos ou financeiros deste estudo.

Esta publicação está sob a licença Creative Commons Atribuição 3.0 não Adaptada.

Para ver uma cópia desta licença, visite http://creativecommons.org/licenses/by/3.0/deed.pt_BR. 